\title{
EDITORIAL
}

\section{Analytical Chemistry at Forensic Institutes}

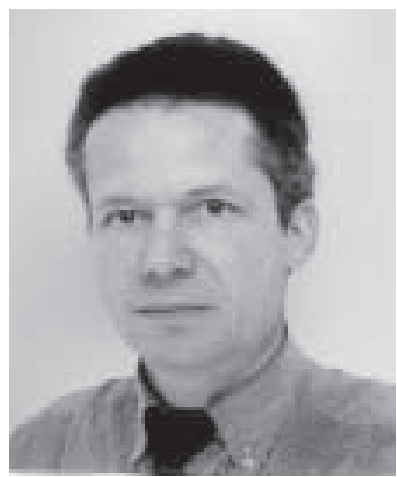

Analytical chemistry at forensic institutes has a respected and long-standing history.

In this issue of CHIMIA we have tried to show the different analytical approaches used today in forensic laboratories. In principle, courts of justice only recognize the results of chemicaltoxicological analyses when they are confirmed by a second independent method. For this reason, gas chromatography coupled with mass spectrometry (GC-MS) is now the method of choice in forensic laboratories. Since LC-MS, another hyphenated technique, cannot yet be used to perform general unknown screenings, this technique remains an excellent complement to GC-MS.

For the determination of ethanol in blood, the aim is not only precision and accuracy but also to provide a test result that allows the forensic medical specialist to deliver an expert opinion. Consumption of psychoactive substances may also impair driving performance. Because of the tremendous diversity of the potential substances, toxicologists have to develop an analytical strategy which allows the detection and quantification of drugs in biological samples.

Morphological, serological, and chemical examination of human hair for forensic and medical purposes was initiated several years ago. A single human hair is sometimes the only remnant at the scene of a crime. In the 1960s, hair analysis was used to evaluate exposure to toxic heavy metals, such as arsenic, lead or mercury. Simultaneously in the United States and West Germany, it was possible to determine various organic drugs in hair at the beginning of the 1980s. At present, hair analysis is almost routinely used as a tool for detection of drug use in forensic science, traffic, and occupational medicine.

DNA typing is a useful tool in a number of situations including the identification of the perpetrators of violent crime such as murder and rape, resolving paternity cases, historical investigations, identifying remains of missing persons or victims of mass disasters, and forensic DNA databases.

Some of the toxicology forensic laboratories are also involved in the analysis of drugs of abuse, e.g. heroin, cocaine and cannabinoids.

In Switzerland, the only accredited laboratory for the analysis of possible doping in sports is located in Lausanne at the Institute of Forensic Medicine. New trends in this field are presented by one of the founders of this laboratory.

Finally two reviews emphasize on the one hand the analysis of a new generation of drugs on the example of gamma-hydroxybutyrate (GHB), and on the other hand, a relatively recent technique capillary electrophoresis (CE).

I would like to extend thanks to all the contributors to this volume for their stimulating work. It is hoped that their reviews will interest a wide spectrum of readers, making perhaps a few converts and stimulating further developments in this interesting and exciting field.

Christian Staub

Institut Universitaire de Médecine Légale

Unité de Toxicologie

9 avenue de Champel

$\mathrm{CH}-1211$ Genève 4

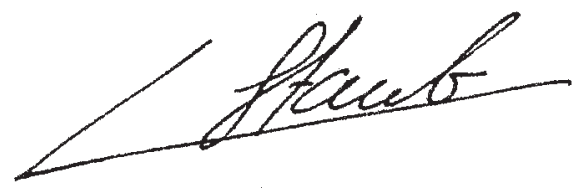

The Editorial Board of CHIMIA expresses its warmest thanks to the coordinating guest editor Dr. Christian Staub for his efforts in the planning and efficient realization of the present attractive overview on 'Analytical Chemistry at Forensic Institutes'. 